18

\title{
Акустодесорбция щелочных металлов и галогенов с однослойного графена: простые оценки
}

\author{
() С.Ю. Давыдов \\ Физико-технический институт им. А.Ф. Иофрфе РАН, \\ Санкт-Петербург, Россия \\ Санкт-Петербургский национальный исследовательский университет \\ информационных технологий, механики и оптики, \\ Санкт-Петербург, Россия \\ E-mail: Sergei_Davydov@mail.ru \\ (Поступила в Редакцию 22 сентября 2016 г.)
}

\begin{abstract}
Для адсорбции атомов щелочных металлов и галогенов на однослойном графене проведены оценки увеличения вероятности термодесорбции под действием волны деформации, или акустодесорбции. Для этого, во-первых, предложено простое аналитическое выражение для энергии адсорбции. Во-вторых, с использованием разработанной ранее $M$-модели адсорбции рассмотрено влияние переменного во времени гидростатического сжатия-растяжения листа графена на заряд адатома и энергию адсорбции. Показано, что для галогенов значения производной энергии адсорбции по деформации на порядок больше, чем для щелочных металлов, а поток десорбируемых атомов максимален при десорбции иода. Для исследования зависимости заряда адатома от деформации использовалось также низкоэнергетическое приближение (НЭП). При этом оценки для щелочных металлов в рамках НЭП показали удовлетворительное согласие с результатами $M$-модели. В рамках НЭП продемонстрировано, что одноосная и гидростатическая деформации приводят к одинаковым по порядку величины эффектам.
\end{abstract}

DOI: 10.21883/FTT.2017.04.44290.356

\section{1. Введение}

Экспериментаторы, имеющие дело с вакуумом, хорошо знают, что если в процессе работы ударить по вакуумной камере, то в системе наблюдается всплеск давления. Это доказывает, что упругая волна деформации, возникающая в стенках камеры, вызывает десорбцию молекул газа, или акустодесорбцию [1-3]. Простая теория акустодесорбции газов с металлических подложек была предложена в работе [4]. Суть эффекта сводится к следующему.

Пусть по поверхности твердотельной подложки $(z=0)$ вдоль оси $x$ распространяется упругая волна со смещениями $u_{0} \cos (k x-\omega \tau)$, где $k-$ волновой вектор, $\omega-$ частота, $\tau-$ время. Предположим, что теплота десорбции $Q$, равная взятой с обратным знаком энергии адсорбции $W$, может быть представлена в виде $Q=Q_{0}+|\Delta Q| \cos (k x-\omega t)$, где $Q_{0}-$ теплота десорбции в отсутствие деформации. Вероятность термодесорбции частицы $\widetilde{f}(Q) \propto \exp (-Q / T)$, где $T$ - температура в энергетических единицах. Перейдем к длинноволновому пределу $k \rightarrow 0$ и будем считать, что в первый полупериод, соответствующий, например, сжатию, можно положить $Q \sim Q_{0}+Q_{1}$, а во второй полупериод, соответствующий растяжению, $Q \sim Q_{0}-Q_{1}$. Тогда вероятность десорбции за период колебаний есть $\widetilde{f}(Q) \sim f_{0}\left(Q_{0}\right) \operatorname{ch}\left(Q_{1} / T\right)$. Отсюда следует, что $\tilde{f}(Q)>f_{0}\left(Q_{0}\right)$. В настоящей работе рассмотрено влияние акустического возмущения на адсорбционные характеристики однолистного графена. Насколько известно автору, такая задача до сих пор не ставилась.

\section{2. Оценки энергии адсорбции}

Общие выражения для энергии адсорбции $W$ атома на однолистном графене приведены в работе [5]. Поскольку эти выражения достаточно громоздки, а целью настоящей работы являются лишь оценки (в лучшем случае полуколичественные), ограничимся здесь упрощенной схемой определения $W$. Как известно [6], энергия адсорбции $W$ может быть представлена в виде суммы ионной $W_{i}$ и металлической $W_{m}$ составляющих. Одной из основных микроскопических характеристик адсорбционной системы является переход заряда $\Delta n$ между адатомом и подложкой [6]. Если одноэлектронное состояние адсорбируемого атома изначально (до адсорбции) заполнено, то $\Delta n=1-n_{a}$, где $n_{a}$ - число заполнения адатома. При этом имеет место переход электронов с адатома в подложку, и заряд адатома равен $Z_{a}=1-n_{a}$. Если же одноэлектронное состояние адсорбируемого атома изначально пусто, то $\Delta n=n_{a}$, электроны переходят из подложки на адатом, и $Z_{a}=-n_{a}$. В общем случае число заполнения $n_{a}=n_{b}+n_{l}$, где первое и второе слагаемые представляют собой вклады зонных и локальных состояний соответственно [6].

Считая графен идеальной проводящей плоскостью, ионную составляющую энергии адсорбции можно было бы оценить с помощью классического выражения $W_{i}^{0} \sim-\left(Z_{a} e\right)^{2} / 4 d$, где $d-$ длина адсорбционной связи, $e$ - величина заряда электрона [6]. Учтем, однако, явным образом, что при удалении адатома от листа графена его заряд стремится к нулю, для чего положим $Z_{a}(z)=Z_{a} \exp (-\kappa(z-d))$, где $\kappa-$ обратная 
длина затухания заряда. Интегрируя по $z$ от $(d$ до $\infty)$ силу взаимодействия заряда $Z_{a}(z)$ с его изображением, получим

$$
W_{i} \approx-\left(Z_{a} e\right)^{2} D / 4 d
$$

где $D=1+2 \kappa d \exp (2 \kappa d) \operatorname{Ei}(-2 \kappa d), \quad \mathrm{Ei}-$ интегральная показательная функция. Положим $\kappa=K$, где $K=2 \pi / 3 \sqrt{3} a$ - вектор точки Дирака, $a=1.42 \AA-$ равновесное расстояние между ближайшими атомами углерода в графене. Учитывая, что для рассматриваемых нами далее случаев $2 K d>1$, положим для простоты $D \approx 1 / 2 K d[7]$.

Для оценки металлической составляющей энергии адсорбции $W_{m}$ воспользуемся соотношением неопределенности Гейзенберга $\Delta p \Delta r \sim \hbar$, где $\Delta p$ и $\Delta r-$ неопределенности импульса и координаты электрона, $\hbar$ - приведенная постоянная Планка. Считая кинетическую энергию электрона (дырки) в свободном атоме величиной порядка $\hbar^{2} / 2 m r_{a}^{2}\left(r_{a}-\right.$ атомный радиус адсорбируемого атома), а в адсорбированном состоянии - порядка $\hbar^{2} / 2 m d^{2}$, имеем выигрыш в энергии на один электрон $\sim\left(\hbar^{2} / 2 m\right)\left(r_{a}^{-2}-d^{-2}\right)$, где $m-$ масса электрона. Учитывая, что в подложку переходят $\left|Z_{a}\right|$ электронов (дырок), ${ }^{1}$ получим

$$
W_{m} \approx-\left(\hbar^{2}\left|Z_{a}\right| / 2 m\right)\left(r_{a}^{-2}-d^{-2}\right) .
$$

В дальнейшем, как и в $[8,9]$, положим $d=r_{a}+r_{\mathrm{C}}$, где $r_{\mathrm{C}}$ - атомный радиус углерода. Таким образом, для оценки энергии адсорбции $W$ достаточно определить значение $Z_{a}$.

В работе [8] была предложена простая модель ( $M$-модель) плотности состояний однослойного графена, позволившая вполне удовлетворительно описать переход заряда при адсорбции атомов водорода, щелочных металлов и галогенов [9]. Взяв $Z_{a}$ из [9], ${ }^{2} r_{a}$ для щелочных металлов и $r_{\mathrm{C}}=0.77 \AA$ из [10], $r_{a}$ для галогенов из [11] (по Полингу), получим оценки значений $W_{i}, W_{m}$ и $W$, представленные в табл. 1 и 2.

Из табл. 1 следует, что энергии $W_{i}, W_{m}$ и $W$ существенно убывают по абсолютной величине в ряду $\mathrm{Li} \rightarrow \mathrm{Cs}$, что объясняется ростом длины адсорбционной связи $d$. Заряды адатомов $Z_{a}$ при переходе от Li к Cs незначительно возрастают. Сопоставление полученных нами значений $d$ с результатами расчетов $[12-14,16]$ демонстрирует, на наш взгляд, приемлемость принятой нами оценки $d=r_{a}+r_{\mathrm{C}}$. С другой стороны, полученные нами значения $Z_{a}$ для адатомов $\mathrm{Na}$ и $\mathrm{K}$ заметно превышают заряды, вычисленные в $[12,13]$, тогда как значение для заряда адатома Cs практически совпадает с результатом работы [14]. Подчеркнем, что разброс значений $Z_{a}$, приводимых в [12,13], также нельзя считать малым. Этот вывод относится не только к работам разных авторов: так, например, рассчитанные в [12] двумя

\footnotetext{
${ }^{1}$ Вообще говоря, энергия $W_{m}$ отлична от нуля и при $Z_{a}=0$. Мы полагаем здесь, что оценка (2) может быть применена для адсорбционных систем со сравнительно большим переходом заряда.

2 Выбираем значения $Z_{a}$, отвечающие расчетам по приближенным формулам для $\sigma$-связи $p$-орбиталей адатома и атома углерода.
}

Таблица 1. Длина адсорбционной связи $d,(\AA)$, заряд адатома $Z_{a}$, ионный $W_{i}$ и металлический $W_{m}$ вклады в энергию адсорбции и полная энергия адсорбции $W(\mathrm{eV})$ щелочных

\begin{tabular}{|c|c|c|c|c|c|}
\hline Параметр & $\mathrm{Li}$ & $\mathrm{Na}$ & K & $\mathrm{Rb}$ & Cs \\
\hline$d$ & 2.34 & 2.63 & 3.13 & 3.25 & 3.39 \\
\hline$Z_{a}$ & 0.89 & 0.91 & 0.96 & 0.96 & 0.97 \\
\hline$-W_{i}$ & 0.31 & 0.25 & 0.20 & 0.18 & 0.18 \\
\hline$-W_{m}$ & 0.76 & 0.50 & 0.28 & 0.25 & 0.22 \\
\hline$-W$ & 1.07 & 0.75 & 0.48 & 0.43 & 0.40 \\
\hline$d$ & 2.23 & 2.70 & 2.99 & & \\
\hline$Z_{a}$ & 0.90 & 0.73 & 0.76 & & \\
\hline $\begin{array}{l}-W \\
{[12]}\end{array}$ & 1.10 & 0.46 & 0.80 & & \\
\hline$d$ & & 2.69 & 2.97 & & \\
\hline$Z_{a}$ & & 0.52 & 0.62 & & \\
\hline $\begin{array}{l}-W \\
{[13]}\end{array}$ & & 0.46 & 0.79 & & \\
\hline $\begin{array}{c}d \\
Z_{a} \\
{[14]}\end{array}$ & & & & & $\begin{array}{l}3.01 \\
0.95\end{array}$ \\
\hline $\begin{array}{c}d \\
-W \\
{[15]}\end{array}$ & $\begin{array}{l}1.64 \\
1.60\end{array}$ & & & & \\
\hline$d$ & 2.02 & 2.67 & 2.65 & 2.77 & \\
\hline $\begin{array}{l}-W \\
{[16]}\end{array}$ & 1.09 & -0.30 & -1.93 & -2.647 & \\
\hline
\end{tabular}
металлов на однослойном графене

Примечание. Данные по $Z_{a}$ и $W$ взяты из [12] для расчета по плотности состояний и для $H$-позиции адатома (hollow site) соответственно.

Таблица 2. Длина адсорбционной связи $d(\AA)$, заряд адатома $Z_{a}$, ионный $W_{i}$ и металлический $W_{m}$ вклады в энергию адсорбции и полная энергия адсорбции $W(\mathrm{eV})$ галогенов на однослойном графене

\begin{tabular}{c|c|c|c|c}
\hline Параметр & $\mathrm{F}$ & $\mathrm{Cl}$ & $\mathrm{Br}$ & $\mathrm{I}$ \\
\hline$d$ & 1.41 & 1.76 & 1.66 & 2.10 \\
$-Z_{a}$ & 0.53 & 0.54 & 0.52 & 0.43 \\
$-W_{i}$ & 0.47 & 0.20 & 0.21 & 0.09 \\
$-W_{m}$ & 2.14 & 1.43 & 1.78 & 0.55 \\
$-W$ & 2.61 & 1.63 & 1.99 & 0.64 \\
& & & & \\
$d$ & 1.47 & 2.71 & 3.01 & \\
& $(1.37,1.87)$ & $(2.54,2.56)$ & $(2.78)$ & \\
$-Z_{a}$ & $0.24,0.29$ & $0.22,0.22$ & $0.05,0.05$ & \\
& $(0.59,0.22)$ & $(0.42)$ & $(0.34)$ & \\
$-W$ & 2.41 & 1.48 & 1.18 & \\
{$[17]$} & $(2.71,2.90)$ & $(0.89,1.05$, & $(0.28,1.00)$ & \\
& & $1.10,1.30)$ & &
\end{tabular}

Примечание. Два значения $Z_{a}$ отвечают различным способам расчета, в скобках представлены результаты работ, ссылки на которые приведены в [17]. 
методами значения $Z_{a}$ различаются приблизительно в 2 раза (см. табл. III в [12]). Следует отметить также, что эффективный заряд является плохо определенным (illdefined) параметром. Полученное нами для Li значение энергии адсорбции $W$ хорошо согласуется с результатами расчетов $[12,16]$. Однако для $\mathrm{Na}$ и $\mathrm{K}$, согласия наших данных с полученными в $[12,13]$ не наблюдается. Нетрудно видеть, однако, и существенные расхождения результатов численных расчетов $[12,13]$ с одной стороны и [16] с другой.

Из табл. 2 следует, что энергии $W_{i}, W_{m}$ и $W$ значительно убывают по абсолютной величине в ряду $\mathrm{F} \rightarrow \mathrm{Br} \rightarrow \mathrm{Cl} \rightarrow \mathrm{I}$. Это объясняется ростом длины адсорбционной связи $d$, заряд же $Z_{a}$ практически одинаков для $\mathrm{F}, \mathrm{Cl}$ и $\mathrm{Br}$, но заметно меньше по абсолютной величине для I. Сравнение полученных нами значений $W$ для адатома фтора с вычисленными в работе [17] показывает хорошее согласие, то же относится и к длинам связи $d$. Этого, однако, нельзя сказать о полученных нами результатах для адатомов хлора и брома. Вновь следует указать и на существенные различия значений $d$, $Z_{a}$ и $W$, вычисленных разными авторами.

В заключение данного раздела отметим, во-первых, что наилучшее согласие имеет место для Li и F, что, на наш взгляд, неудивительно, так как именно эти атомы обладают наименьшими атомными радиусами и поэтому могут с большей достоверностью рассматриваться как точечные заряды в выражении (1). Во-вторых, вычисленные нами энергии связи для галогенов выше, чем для щелочных металлов.

\section{3. Влияние деформации: $M$-модель}

Рассмотрим однородную по координатам $(x, y)$ и осциллирующую во времени деформацию всестороннего (гидростатического) двумерного сжатия-растяжения, вызывающую изменение расстояния между ближайшими атомами углерода в графене, что можно представить в виде $\widetilde{a}(\tau)=a+|\Delta a| \cos \omega \tau$, где $|\Delta a|$ - амплитуда изменения расстояния $a$ между ближайшими соседями (здесь и далее тильда отвечает деформированному состоянию $^{3}$ ). Сведение рассмотрения к столь простой деформации гарантирует, что щель в электронном спектре графена не возникает, форма плотности состояний графена не меняется, а имеет место только сдвиг границ его плотности состояний. Действительно, в соответствии с методом связывающих орбиталей Харрисона [18] энергия перехода электрона между соседними атомами графена $\widetilde{t} \propto \widetilde{a}^{-2}$, так что $\widetilde{t} \approx t(1-\varepsilon)$, где $\varepsilon=2 \Delta a / a-$ параметр деформации.

Будем считать для простоты, что длина адсорбционной связи $d$ от деформации не зависит. Отсюда следует, что не меняется и матричный элемент взаимодействия адатом-подложка $V$. То же относится и к энергии уров-

\footnotetext{
3 Здесь и далее рассматривается только деформация в плоскости графена (in-plane deformation).
}

Таблица 3. M-модель: результаты расчета влияния деформации для адсорбции щелочных металлов на однослойном графене

\begin{tabular}{c|c|c|c|c|c}
\hline Металл & $\partial \widetilde{n}_{b} / \partial \varepsilon$ & $\partial \widetilde{n}_{l} / \partial \varepsilon$ & $\xi_{a}$ & $\partial \widetilde{W} / \partial \varepsilon, \mathrm{eV}$ & $\left|W^{-1}\left(\partial \widetilde{W}^{-1}\right) / \partial \varepsilon\right|$ \\
\hline $\mathrm{Li}$ & -0.17 & 0 & 0.19 & -0.27 & 0.25 \\
$\mathrm{Na}$ & 0.01 & 0 & -0.01 & 0.12 & 0.16 \\
$\mathrm{~K}$ & 0.11 & 0 & -0.11 & 0.07 & 0.15 \\
$\mathrm{Rb}$ & 0.11 & 0 & -0.11 & 0.07 & 0.16 \\
$\mathrm{Cs}$ & 0.11 & 0 & -0.11 & 0.06 & 0.15
\end{tabular}

Таблица 4. $M$-модель: результаты расчета влияния деформации для адсорбции галогенов на однослойном графене

\begin{tabular}{c|r|r|r|c|c}
\hline Галоген & $\partial \widetilde{n}_{b} / \partial \varepsilon$ & $\partial \widetilde{n}_{l} / \partial \varepsilon$ & $\xi_{a}$ & $\partial \widetilde{W} / \partial \varepsilon, \mathrm{eV}$ & $\left|W^{-1}\left(\partial \widetilde{W}^{-1}\right) / \partial \varepsilon\right|$ \\
\hline $\mathrm{F}$ & 1.00 & -0.25 & 1.42 & -4.36 & 1.67 \\
$\mathrm{Cl}$ & -0.17 & -0.23 & -0.93 & 1.69 & 1.04 \\
$\mathrm{Br}$ & -0.45 & -0.20 & -1.25 & 2.76 & 1.39 \\
$\mathrm{I}$ & -0.67 & -0.11 & -1.81 & 1.33 & 2.08
\end{tabular}

ня адатома $\varepsilon_{a}$. Ясно также, что обратную длину затухания заряда при десорбции, которую мы приравняли к $K$, также логично считать константой. С учетом принятых упрощений имеем $\partial \widetilde{W}_{i} / \partial \varepsilon=2 W_{i} \xi_{a}$ и $\partial \widetilde{W}_{m} / \partial \varepsilon=W_{m} \xi_{a}$, где $\xi_{a}=Z_{a}^{-1}\left(\partial \widetilde{Z}_{a} / \partial \varepsilon\right)$. Таким образом, получаем

$$
\partial \widetilde{W} / \partial \varepsilon=\left(2 W_{i}+W_{m}\right) \xi_{a} .
$$

Задача, следовательно, сводится к вычислению параметра $\xi_{a}$, или зависимости заряда адатома от деформации. Имеем $\partial \widetilde{Z}_{a} / \partial \varepsilon=-\partial \widetilde{n}_{a} / \partial \varepsilon$, где число заполнения адатома $\widetilde{n}_{a}=\widetilde{n}_{b}+\widetilde{n}_{l}, \widetilde{n}_{b}-$ вклад зонных состояний, $\tilde{n}_{l}$ - вклад локального состояния, лежащего ниже дна валентной зоны графена $[6,8]$. Воспользовавшись для вычисления $\partial \widetilde{n}_{b} / \partial \varepsilon$ и $\partial \widetilde{n}_{l} / \partial \varepsilon$ результатами работы [8], получим выражения, приведенные в Приложении (пункт 1). Результаты расчетов приведены в табл. 3 и $4 .{ }^{4}$

Из табл. 3 следует, что в случае адсорбции щелочных металлов $\partial \widetilde{Z}_{a} / \partial \varepsilon \approx-\partial \widetilde{n}_{b} / \partial \varepsilon$, так как вклад $\partial \widetilde{n}_{l} / \partial \varepsilon$ исчезающе мал. Согласно (П9) и данным [9], это обстоятельство обусловлено практически нулевым вкладом $n_{i}$ в $n_{a}$. С другой стороны, из табл. 4 следует, что для адсорбции галогенов на однолистном графене вклады $\partial \widetilde{n}_{b} / \partial \varepsilon$ и $\partial \widetilde{n}_{l} / \partial \varepsilon$ сравнимы, так же как и значения $n_{b}$ и $n_{l}$ [9]. Отметим, что величины $\left|\partial \widetilde{Z}_{a} / \partial \varepsilon\right|$ и $|\partial W / \partial \varepsilon|$ для адатомов галогенов более чем на порядок превосходят соответствующие величины для адатомов щелочных металлов. Следует также подчеркнуть особенности производных $\partial \widetilde{n}_{b} / \partial \varepsilon$ для лития и фтора, отличающихся как по величине, так и по знаку от значений этих производных для других элементов I и VII столбцов Периодической системы. В случае адатома Li

\footnotetext{
${ }^{4}$ Для расчета взяты значения параметров $\gamma$ и $\beta$, отвечающие $\sigma$-связи $p$-орбитали атома углерода с $s$ - и $p$-орбиталями адатомов щелочных металлов и галогенов соответственно.
} 
это объясняется высоким значением параметра $\beta \approx 32$, в результате чего основной вклад в $\partial \widetilde{n}_{b} / \partial \varepsilon$ вносит слагаемое $\partial \widetilde{I}_{2} / \partial \varepsilon \approx-0.27$ (см. Приложение, пункт 1 ). Более того, высокое значение параметра $\beta \approx 21$ для $\mathrm{Na}$ приводит к тому, что $\partial \widetilde{n}_{b} / \partial \varepsilon<0.01$. В случае адатома $\mathrm{F}$ малое значение $\beta \approx 0.72$ приводит к аномально большой величине $\partial \widetilde{I}_{12} / \partial \varepsilon \approx 1.08$, определяющей величину производной $\partial \widetilde{n}_{b} / \partial \varepsilon$.

\section{4. Влияние деформации: низкоэнергетическое приближение}

В низкоэнергетическом приближении (НЭП) спектр электронов представляют в виде $\varepsilon_{ \pm}(\mathbf{q})=$ $= \pm(3 t a / 2)|\mathbf{q}|[19]$, где энергия точки Дирака вновь принимается за нуль. Задача об адсорбции в рамках НЭП была рассмотрена в работе [20]. Учитывая всестороннюю деформацию, легко показать, что плотность состояний графена $\widetilde{\rho}(\omega)=2|\omega| / \widetilde{\xi}^{2}$ при $|\omega| \leq \widetilde{\xi}$ и $\widetilde{\rho}(\omega)=0$ при $|\omega|>\widetilde{\xi}$, где $\omega-$ энергетическая переменная, $\widetilde{\xi}=3 \widetilde{t} \widetilde{a} \widetilde{q}_{c} / 2, \widetilde{q}_{c}-$ волновой вектор обрезания. Полагая, как и авторы $[21], \pi \widetilde{q}_{c}^{2}=(2 \pi)^{2} / \widetilde{S}$, где площадь элементарной ячейки $\widetilde{S}=3 \sqrt{3} \widetilde{a}^{2} / 2$, получим $\widetilde{\rho}(\omega)=\rho(\omega(1+2 \varepsilon)), \widetilde{\xi}=\xi(1-\varepsilon)$ и $\xi=\sqrt{2 \pi \sqrt{3}} t$.

При наличии однородной гидростатической деформации зонная составляющая числа заполнения есть

$$
\widetilde{n}_{b}=\frac{1}{\pi} \int_{-\widetilde{\xi}}^{0} d \omega \frac{\widetilde{\Gamma}(\omega)}{\left(\omega-\varepsilon_{a}-\widetilde{\Lambda}(\omega)\right)^{2}+\Gamma^{2}(\omega)},
$$

где полуширина и функция сдвига квазиуровня адатома равны соответственно $\widetilde{\Gamma}(\omega)=\pi V^{2} \widetilde{\rho}(\omega)$ и $\widetilde{\Lambda}(\omega)=\left(2 V^{2} / \widetilde{\xi}^{2}\right) \omega \ln \left[\omega^{2} /\left(\widetilde{\xi}^{2}-\omega^{2}\right)\right], \quad V-$ матричный элемент взаимодействия адатом-графен, уровень Ферми проходит через точку Дирака. Дифференцируя выражение (4) по деформации $\varepsilon$, можно показать (см. Приложение, пункт 2), что

$$
\partial \widetilde{n}_{b} / \partial \varepsilon=2 n_{b}+I
$$

где интеграл $I$ определяется формулой (П10). Далее мы вновь прибегнем к упрощениям и рассмотрим случай, когда величина энергии уровня адатома $\left|\varepsilon_{a}\right| \sim t$, что соответствует адсорбции щелочных металлов $[9,20]$. Тогда, как показано в пункте 2 Приложения, получим $I \approx-(32 / 3 \sqrt{2 \pi \sqrt{3}})\left(V^{4} / t \varepsilon_{a}^{3}\right)$. Отметим, что $\partial \widetilde{n}_{l} / \partial \varepsilon$ попрежнему определяется формулой (П9).

Результаты оценок приведены в табл. 5. Сопоставление с табл. 3 показывает, что по порядку величины результаты, полученные в рамках НЭП и $M$-модели, согласуются достаточно удовлетворительно. ${ }^{5}$ При переходе от $\mathrm{Li}$ к $\mathrm{Cs}$ значение $\xi_{a}$ уменьшается и меняет знак. Наибольшее расхождение (как по величине $\xi_{a}$,

\footnotetext{
${ }^{5}$ В отсутствие деформации сходства и различия результатов НЭП и $M$-модели обсуждаются в работе [20].
}

Таблица 5. Низкоэнергетическое приближение: результаты расчета влияния деформации для адсорбции щелочных металлов на однослойном графене

\begin{tabular}{c|c|c|c|c}
\hline Металл & $Z_{a}[20]$ & $\partial \widetilde{n}_{b} / \partial \varepsilon$ & $\partial \widetilde{n}_{l} / \partial \varepsilon$ & $\xi_{a}$ \\
\hline $\mathrm{Li}$ & 0.83 & -0.28 & -0.10 & 0.46 \\
$\mathrm{Na}$ & 0.89 & -0.21 & -0.07 & 0.31 \\
$\mathrm{~K}$ & 0.93 & 0.05 & -0.01 & -0.04 \\
$\mathrm{Rb}$ & 0.94 & 0.07 & 0 & -0.07 \\
$\mathrm{Cs}$ & 0.95 & 0.07 & 0 & -0.07
\end{tabular}

так и по знаку) имеет место для $\mathrm{Na}$. Мы здесь не приводим значения $\partial \widetilde{W} / \partial \varepsilon$ и $\left|W^{-1}(\partial \widetilde{W} / \partial \varepsilon)\right|$, которые можно легко получить путем пересчета приведенных в табл. 3 результатов.

Отметим, что в рамках НЭП нетрудно в принципе учесть любой вид плоской деформации листа графена, вводя в расчет соответствующий закон дисперсии электронов [22,23]. В Приложении (пункт 3) показано, что одноосная деформация по порядку величины дает то же значение относительного изменения заряда адатома $\xi_{a}$, что и гидростатическая деформация.

\section{5. Заключение}

Согласно $M$-модели, увеличение вероятности десорбции под действием переменной во времени деформации сжатия-растяжения определяется функцией $f_{1} \equiv \operatorname{ch}(\varepsilon|\partial \widetilde{W} / \partial \varepsilon| / T)$, которая убывает в ряду $\mathrm{F} \rightarrow \mathrm{Br} \rightarrow \mathrm{Cl} \rightarrow \mathrm{I} \rightarrow \mathrm{Li} \rightarrow \mathrm{Na} \rightarrow \mathrm{K}, \mathrm{Rb} \rightarrow \mathrm{Cs}$. Для эксперимента, однако, важно не только значение $f_{1}$, но и вероятность термодесорбции $f_{0}=\exp (W / T)$ в отсутствие деформации. Ясно, что максимальное значение вероятности слета адчастиц $\widetilde{f}=f_{0} f_{1}$ наблюдается при максимальном значении отношения $\left|W^{-1}(\partial \widetilde{W} / \partial \varepsilon)\right|$. Как следует из табл. 3 и 4, это отношение для галогенов на порядок больше, чем для щелочных металлов, а среди галогенов максимально для адатомов иода. Выводы $M$-модели для случая адсорбции щелочных металлов были проверены в рамках НЭП. В том же приближении было показано, что одноосная и гидростатическая деформации приводят к одинаковым по порядку величины эффектам.

Следует отметить, что при расчете значений $\partial \widetilde{Z}_{a} / \partial \varepsilon$ мы, как и в работах $[8,9]$, не учитывали влияния температуры. В [24], однако, показано, что роль температуры существенна только в случае близости уровня адатома $\varepsilon_{a}$ к точке Дирака. Как следует из [9], для всех рассмотренных здесь случаев (за исключением, возможно, брома) уровень $\varepsilon_{a}$ достаточно удален от точки Дирака.

Итак, в настоящей работе мы предложили простое выражение для энергии адсорбции и влияния на эту энергию всестороннего сжатия-растяжения. Это позволило нам получить порядковые оценки увеличения термоде- 
сорбции за счет переменной во времени деформации и предсказать, что максимальный поток десорбируемых атомов будет наблюдаться при адсорбции иода. Все эти выводы, естественно, требуют экспериментальной проверки.

\section{Приложение}

1. Основным параметром $M$-модели является безразмерная константа связи $\tilde{\gamma}=\widetilde{\rho}_{m} V^{2} / \widetilde{t}$, которая в силу принятых нами упрощений может быть записана как $\tilde{\gamma} \approx \gamma(1+2 \varepsilon)$. Будем считать $\widetilde{\eta}_{a 1,2}=\eta_{a 1,2}$ (см. подробнее [8]), так как энергия уровня адатома $\varepsilon_{a}$ и произведения $\gamma a_{1,2}$ (по самой сущности схемы определения чисел $a_{1,2}$ ) от деформации не зависят. ${ }^{6}$ Тогда $\widetilde{\beta}=4 \pi \widetilde{\gamma} / \widetilde{\eta}_{a 1}^{2}=\beta$ и $\widetilde{q}=\left|\widetilde{\eta}_{a 1}\right|\left(1+\beta^{2}\right)^{1 / 4} / 2 \approx q(1+\varepsilon)$.

Зонный вклад в число заполнения адатома $n_{b}=\widetilde{I}_{11}+\widetilde{I}_{12}+\widetilde{I}_{13}$, где общий вид интегралов $I_{i j}$ (в отсутствие деформации) и аналитические выражения для них приведены в [8]. Для расчета изменения энергии адсорбции под действием деформации нам нужны значения $\partial n_{b} / \partial \varepsilon$. В результате получаем

$$
\begin{gathered}
\frac{\partial \widetilde{I}_{11}}{\partial \varepsilon} \approx-\frac{\gamma}{2}\left(\frac{3\left(6+\eta_{a 1}\right)}{(\pi \gamma)^{2}+9\left(3+\eta_{a 1}\right)^{2}}-\frac{\left(2+\eta_{a 1}\right)}{(\pi \gamma)^{2}+\left(1+\eta_{a 1}\right)^{2}}\right), \\
-\operatorname{sgn}\left(\eta_{a 1}\right) 4 \pi\left(1+\beta^{2}\right)^{1 / 4} \frac{\partial \widetilde{I}_{12}}{\partial \varepsilon} \approx \sin (a / 2) \\
\times\left(\frac{\partial \ln \widetilde{g}_{1-}}{\partial \varepsilon}+\frac{\partial \ln \widetilde{g}_{3+}}{\partial \varepsilon}-\frac{\partial \ln \widetilde{g}_{1+}}{\partial \varepsilon}-\frac{\partial \ln \widetilde{g}_{3-}}{\partial \varepsilon}\right) \\
+2 \cos (\alpha / 2)\left(\frac{\partial \widetilde{H}_{3}}{\partial \varepsilon}-\frac{\partial \widetilde{H}_{1}}{\partial \varepsilon}\right),
\end{gathered}
$$

где

$$
\begin{gathered}
\partial \widetilde{g}_{N \pm} / \partial \varepsilon=\left(N+\eta_{a 1} / 2\right) \eta_{a 1} \pm 4 q \cos (\alpha / 2)\left(N+\eta_{a 1} / 2\right)+2 q, \\
\partial \widetilde{H}_{N} / \partial \varepsilon=(\partial \widetilde{h} / \partial \varepsilon) /\left(1+h_{N}^{2}\right), \\
\frac{\partial \widetilde{h}_{N}}{\partial \varepsilon}=\frac{\left(N+\eta_{a 1} / 2\right) \eta_{a 1}-2 q^{2}}{2 q \sin (\alpha / 2)\left(N+\eta_{a 1} / 2\right)} \\
-h_{N}\left[1+\left(N+\eta_{a 1} / 2\right) \eta_{a 1} / 2\right]
\end{gathered}
$$

и $N=1,3$ (здесь мы игнорируем экзотические частные случаи, когда учет деформации меняет знаки функции Хэвисайда, см. [8]);

$$
\frac{\partial \widetilde{I}_{2}}{\partial \varepsilon} \approx-2 \frac{(\pi \gamma)^{2}-1}{(\pi \gamma)^{2}+1} I_{2}+\frac{\gamma}{2\left(1+(\pi \gamma)^{2}\right)}\left(A+\operatorname{sgn}\left(\eta_{a 2}\right) B,\right)
$$

${ }^{6}$ В $[19,22]$ показано, что работа выхода графена в приближении взаимодействия ближайших соседей под влиянием деформации не меняется. Отсюда следует, что энергия точки Дирака и, следовательно, энергия уровня адатома остаются постоянными. где

$$
\begin{aligned}
A= & -\frac{2 \eta_{a 2}^{2}}{\left(1+\eta_{2 a}\right)^{2}+(\pi \gamma)^{2}}\left(\frac{\eta_{a 2}\left(1+\eta_{a 2}\right)+4(\pi \gamma)^{2}}{\eta_{a 2}^{2}}\right), \\
B= & -\frac{4}{\pi \gamma}\left(\operatorname{arctg} \frac{1}{\pi \gamma}-\operatorname{arctg} \frac{1+\eta_{a 2}+(\pi \gamma)^{2}}{\left|\eta_{a 2}\right| \pi \gamma}\right) \\
& -2\left(\frac{2}{(\pi \gamma)^{2}+1}+\frac{\left|\eta_{a 2}\right|\left((\pi \gamma)^{2}-2\right)}{\left(\eta_{a 2} \pi \gamma\right)^{2}+1}\right) .
\end{aligned}
$$

Определим теперь значение $\partial \widetilde{n}_{l} / \partial \varepsilon$. Поскольку положение локального уровня определяется уравнением $x-\eta_{a}-(1+\varepsilon) \lambda(x) \approx 0$, а число его заполнения дается выражением $\widetilde{n}_{l} \approx|1-(1+\varepsilon)(\partial \lambda / \partial x)|_{x_{l}}^{-1}$, легко показать, что

$$
\partial \widetilde{n}_{l} / \partial \varepsilon \approx-n_{l}\left(1-n_{l}\right) .
$$

2. Интегрируя выражение (4), получим $\partial \widetilde{n}_{b} / \partial \varepsilon=$ $=2 n_{b}-\xi \rho_{a}(-\xi)+I$, где интеграл $I$ имеет вид

$I=\frac{4}{\pi} \int_{-\xi}^{0} d \omega \Gamma(\omega) \frac{\left(\omega-\varepsilon_{a}-\Lambda(\omega)\right)[\Lambda(\omega)+C(\omega)]+\Gamma^{2}(\omega)}{\left[\left(\omega-\varepsilon_{a}-\Lambda(\omega)\right)^{2}+\Gamma^{2}(\omega)\right]^{2}}$

и $C(\omega)=2 V^{2} \omega /\left(\xi^{2}-\omega^{2}\right)$. При этом было учтено, что $\partial \widetilde{\Gamma}(\omega) / \partial \varepsilon=2 \Gamma(\omega)$ и $\partial \widetilde{\Lambda}(\omega) / \partial \varepsilon=2[\Lambda(\omega)+C(\omega)]$. При $\omega \rightarrow-\xi$ функция сдвига $\Lambda(\omega) \rightarrow-\infty$ и $\rho_{a}(-\xi) \rightarrow 0$, откуда и получаем формулу (5).

Рассмотрим $\left|\varepsilon_{a}\right| \sim t$, что отвечает адсорбции атомов щелочных металлов. Можно показать, что основной вклад в интеграл вносит область малых энергий (окрестность точки Дирака). Поскольку $\Gamma(\omega)=2 \pi V^{2}|\omega| / \xi^{2}$ и при $\omega \rightarrow 0$ имеем $\widetilde{\Lambda}(\omega) \rightarrow\left(2 V^{2} / \xi^{2}\right)$ $\times \omega \ln \left(\omega^{2} / \xi^{2}\right), C(\omega) \rightarrow\left(2 V^{2} / \xi^{2}\right) \omega$, подставляя эти значения в $(П 10)$, получим $I \approx-(32 / 3 \sqrt{2 \pi \sqrt{3}})\left(V^{4} / t \varepsilon_{a}^{3}\right)$.

3. В работах $[22,23]$ показано, что влияние одноосной деформации $\varepsilon$ на электронный спектр графена в рамках НЭП описывается выражением

$$
\widetilde{\varepsilon}(\mathbf{q})= \pm(3 t a / 2) \sqrt{(1+2 \lambda \varepsilon)^{2} q_{x}^{2}+(1-2 \lambda \nu \varepsilon)^{2} q_{y}^{2}}
$$

где $v-$ коэффициент Пуассона, $\lambda-$ отрицательное число порядка единицы и знаки „плюс“ и „минус“ относятся к зоне проводимости и валентной зоне соответственно. Записывая плотность состояний деформированного графена в виде $\widetilde{\rho}(\omega, \mathbf{q})=s / \pi\left[(\omega-\widetilde{\varepsilon}(\mathbf{q}))^{2}+s^{2}\right]$, где $s=0^{+}$, для энергетической плотности состояний имеем

$$
\widetilde{\rho}(\omega)=C \iint_{\mathrm{DBZ}} d q_{x} d q_{y} \widetilde{\rho}(\omega, \mathbf{q}),
$$

где интегрирование идет по деформированной зоне Бриллюэна (DBZ), C - нормировочная константа. Перейдем к эллиптическим координатам $(r, \varphi)$, полагая $q_{x}=a r \cos \varphi, \quad q_{y}=b r \sin \varphi, \quad$ где $\quad a=(1+2 \lambda \varepsilon)^{-1}$, $b=(1-2 \lambda \varepsilon)^{-1}$. $\quad$ Тогда получаем $\quad \widetilde{\rho}(\omega)=2|\omega| / \widetilde{\xi}^{2}$ 
при $\quad|\omega| \leq \widetilde{\xi} \quad$ и $\quad \widetilde{\rho}(\omega)=0 \quad$ при $\quad|\omega|>\widetilde{\xi}, \quad$ где $\widetilde{\xi} \approx[1+\lambda(1-v) \varepsilon] \xi$ и $\xi=\sqrt{2 \pi \sqrt{3}} t$. Таким образом, $\widetilde{\rho}(\omega)[1-2 \lambda(1-v) \varepsilon] \rho(\omega)$. Так как для гидростатической деформации $\widetilde{\rho}(\omega)=(1+2 \varepsilon) \rho(\omega), \lambda<0$ и $|\lambda| \sim 1$ [23], $v \approx 0.14$ (графит), приходим к выводу, что одноосная деформация по порядку величины дает то же значение относительного изменения заряда адатома $\xi_{a}$, что и гидростатическая деформация.

\section{Список литературы}

[1] D.R. Denison. Vac. Sci. Technol. 6, 214 (1969).

[2] C. Krishar, D. Lichtman. Phys. Lett. A 44, 99 (1973).

[3] C. Krishar, D. Lichtman. Jpn. J. Appl. Phys. 13 (Suppl. 2-2), 469 (1974).

[4] С.Ю. Давыдов, В.И. Марголин. Поверхность 8, 5 (1983).

[5] С.Ю. Давыдов. ФТТ 53, 2414 (2011).

[6] С.Ю. Давыдов. Теория адсорбции: метод модельных гамильтонианов. Изд-во СПбГЭТУ „ЛЭТИ“, СПб (2013). 235 c. twirpx.com/file/1596114/

[7] Е. Янке, Ф. Эмде, Ф. Лёш. Специальные функции. Формулы, графики, таблицы. Наука, М. (1977). 341 с.

[8] С.Ю. Давыдов, Г.И. Сабирова. ФТТ 53, 608 (2011).

[9] С.Ю. Давыдов, Г.И. Сабирова. Письма в ЖТФ 37, 11, 51 (2011).

[10] Физические величины. Справочник / Под ред. И.С. Григорьева, Е.3. Мейлихова. Энергоатомиздат, М. (1991). $1232 \mathrm{c}$.

[11] Краткий справочник физико-химических величин / Под ред. К.П. Мищенко, А.А. Равделя. Химия, Л. (1974). 200 с.

[12] K.T. Chan, J.B. Neaton, M.L. Cohen. Phys. Rev. B, 77, 235430 (2008).

[13] X. Liu, C.Z. Wang, Y.X. Yao, W.C. Lu, M. Hupalo, M.C. Tringides. Phys. Rev. B 83, 235411 (2011).

[14] J.-H. Parq, J. Yu, Y.-K. Kwon, G. Kim. Phys. Rev. B 82, 193406 (2010).

[15] M. Khamtha, N.A. Cordero, L.M. Molina, J.A. Alonso, L.A. Girifalco. Phys. Rev. B 70, 125422 (2004).

[16] P.V.C. Medeiros, F. de Brito Mota, A.J.S. Mascarenhas, C.M.C. de Castilho. Nanotechnology 21, 115701 (2010).

[17] D.B. Karki, N.P. Adhikari. Int. J. Mod. Phys. B 28, 1450141 (2014).

[18] У. Харрисон. Электронная структура и свойства твердых тел. Мир, М. (1983). Т. 1, 382 с.

[19] A.H. Castro Neto, F. Guinea, N.M.R. Peres, K.S. Novoselov, A.K. Geim. Rev. Mod. Phys. 81, 109 (2009).

[20] С.Ю. Давыдов, О.В. Посредник. ФТТ 57, 1654 (2015).

[21] N.M.R. Peres, F. Guinea, A.H. Castro Neto. Phys. Rev. B 73, 125411 (2006)

[22] F.M.D. Pellegrino, G.G.N. Angilella, R. Pucci. Phys. Rev. B 84, 195404 (2011).

[23] B. Wang, Y. Wang, Y. Liu. Funct. Mater. Lett. 8, 1530001 (2015).

[24] С.Ю. Давыдов. ЖТФ 86, 7, 145 (2016). 